\title{
Unfolding of antiferromagnetic phases and multicritical points in a two-orbital model for uranium compounds under pressure and magnetic field
}

\author{
S. G. Magalhaes* \\ Instituto de Física, Universidade Federal do Rio Grande do Sul, 91501-970 Porto Alegre, RS, Brazil \\ A. C. Lausmann and E. J. Calegari \\ Departamento de Física-UFSM, 97105-900, Santa Maria, RS, Brazil \\ P. S. Riseborough \\ Physics Department, Temple University, Philadelphia, Pennsylvania 19122, USA
}

(Received 29 August 2019; revised manuscript received 8 January 2020; accepted 22 January 2020; published 6 February 2020)

\begin{abstract}
We investigate the occurrence of multicritical points under pressure and magnetic field in a model that describes two $5 f$ bands (of either $\alpha$ or $\beta$ characters), which hybridize with a single itinerant conduction band. The $5 f$ electrons interact through Coulomb and exchange terms. The antiferromagnetic (AF) order parameter is a Néel vector, which is assumed to be fixed by an Ising anisotropy. The applied magnetic field is transverse to the anisotropy axis. Without field, our results for the temperature-pressure phase diagram show that at low temperatures a first-order phase transition occurs between two distinct antiferromagnetic phases, $\mathrm{AF}_{1}$ and $\mathrm{AF}_{2}$, as the pressure is increased. The two phases are characterized by the gaps of bands $\alpha$ and $\beta$ given by $\Delta_{\alpha}$ and $\Delta_{\beta}$, respectively. The $\mathrm{AF}_{1}$ phase occurs when $\Delta_{\beta}>\Delta_{\alpha}>0$, while in the $\mathrm{AF}_{2}$ phase the gaps satisfy $\Delta_{\alpha}>\Delta_{\beta}>0$. The application of a magnetic field produces a drastic change in the phase diagram. The $\mathrm{AF}_{1}$ and $\mathrm{AF}_{2}$ phases separate, with the latter acquiring a dome shape that is eventually suppressed for large values of the applied field. The evolution of the phase diagram under pressure, without and with magnetic field, shows the presence of multicritical points. Our results show that the evolution of these multicritical points by the simultaneous application of pressure and magnetic field is also drastic, with the suppression of some multicritical points and the emergence of others. We believe that these results may have relevance for the growing field of multicritical points (classical and quantum) in the physics of uranium compounds.
\end{abstract}

DOI: 10.1103/PhysRevB.101.064407

\section{INTRODUCTION}

The uniqueness of the $5 f$-electrons' physics is due to their dual localized-delocalized character [1]. Uranium compounds display a variety of quantum states of matter, such as magnetism (localized and itinerant) [2], superconductivity [3], and the enigmatic hidden order [4,5]. These phases can be tuned by pressure (hydrostatic or chemical) and magnetic field. The plethora of distinct types of ordering hosted by uranium compounds makes these systems a natural ground for the appearance of multicritical points. Likewise, the presence of a specific kind of multicritical point could be useful in the clarification of an unconventional symmetry breaking that may exist in uranium compounds. Multicritical points driven by thermal fluctuations might evolve into quantum multicritical points [6] as pressure or magnetic field increase [7], leading to deviations of Fermi liquid behavior $[8,9]$.

The presence of multiple $5 f$ orbitals in uranium compounds has important consequences for their physics $[10,11]$. The underscreened Anderson lattice model (UALM) that we use consists of two degenerate narrow $5 f$ bands (denoted by $\chi=\alpha, \beta)$, which acquire an itinerant character by direct hop-

*Corresponding author: sgmagal@gmail.com ping between neighboring $5 f$ shells. The resulting two bands are also hybridized with a single itinerant conduction band. Their interaction is composed of the Coulomb interaction between electrons in the same $5 f$ bands and the Hund's rule exchange interaction between electrons in distinct bands. This model considers the ground state as a triplet $(S=1)$, which can be related via a Schrieffer-Wolff transformation with the underscreened Kondo lattice [12], which has successfully described the coexistence of the Kondo effect and ferromagnetism found in uranium monochalcogenides [13]. For the purposes of this work, which does not deal with the Kondo effect, the UALM is important because it can describe not only the antiferromagnetic ordering observed in the uranium pnictides [14-16] and $\mathrm{UIrSi}_{3}$ [17] but has also been proposed to describe the hidden order phase of the $\mathrm{URu}_{2} \mathrm{Si}_{2}$ [18].

The UALM is suited for the investigation of time-reversal symmetry breaking as a source of unfolding of phases and multicritical points. The Hund's rule exchange interaction term is essential because it can make the model spinrotationally invariant [19] and opens distinct routes to longrange ordering. For example, a phase transition can be driven by the spin-flip part of Hund's rule exchange interaction, breaking spin-rotational and space-translational symmetries but preserving the time-reversal symmetry. Consequently, a novel ordered state can be stabilized in which there is 
spontaneous $5 f$ inter-band mixing, which does not involve magnetic order. This novel type of long-range order has been proposed as describing the hidden order phase in $\mathrm{URu}_{2} \mathrm{Si}_{2}$ [18]. The interaction terms can also produce conventional antiferromagnetic long-range order in the UALM. The (here called) intraorbital antiferromagnetic phases (IOAF) appear below a magnetic phase transition at which spin-rotational, space-translational, and time-reversal symmetries are broken. This transition gives rise to not one but two distinct competing IOAF phases, which have spin gaps at the same ordering wave vector. Therefore, in the transition between the two IOAF phases, no further symmetries are broken.

In this work, we investigate the temperature-pressuremagnetic field phase diagram of the IOAF phases within a mean-field approximation. The objective is to explore the UALM, and to describe the unfolding of an itinerant antiferromagnetic phase and the subsequent competition between the two unfolded different antiferromagnetic phases. In particular, we focus on multicritical points that can appear from that competition, and how they evolve with pressure and field. The symmetry contained in the model that can be broken (as the time reversal one) will be reflected in the parity properties of the order parameters, which condition the emergence of multicritical points. We stress that the subject of multicritical points can be connected to real uranium compounds. For instance, a bicritical point was found in $\mathrm{URu}_{2} \mathrm{Si}_{2}$ [4] when pressure is applied and tricritical (TCP) points were found in $\mathrm{USb}_{2}$ [20], $\mathrm{UN}$ [21], $\mathrm{UAu}_{2} \mathrm{Si}_{2}$ [22], and $\mathrm{URu}_{2} \mathrm{Si}_{2}$ [23] when a magnetic field is applied. However, the simultaneous effect of pressure and magnetic field on multicritical points has received little theoretical attention (for an exception, see Ref. [24]) in the physics of uranium compounds.

We assume that the bandwidth $W$ can be varied by the application of pressure while the hybridization, the Coulomb, and the Hund's rule interactions remain constant. We also make the following assumptions. (i) The hybridization matrix elements are $k$ independent. Consequently, one may transform the basis of the $5 f$ states into a new basis in which a linear combination of $f$ orbitals hybridize and the remaining orthogonal states do not. The asymmetric hybridization breaks the symmetry between the $5 f$ bands; thus intraband nesting may occur simultaneously for both bands. However, when $W$ increases, one band may depart from the perfect nesting condition and, hence, have a reduced moment. Ultimately, above some value of $W$, both bands might not satisfy the nesting condition and the material might become nonmagnetic. (ii) The IOAF has a Néel order parameter that is fixed by an Ising-like anisotropy. This assumption introduces a magnetic anisotropy that is observed in some uranium compounds [25-27]. Consequently, there are two types of field effects in the IOAF $[28,29]$. For a field aligned with the easy axis, the Zeeman splitting between the spin-up and spin-down IOAF subbands increases as the field increases. Meanwhile, for a field along a perpendicular direction, there is a spin-dependent momentum shift of the IOAF bands. In both cases, the nesting condition may no longer be satisfied. We chose the latter possibility due to its spin-flipping effects.

In the case of two distinct IOAF (denoted as $\mathrm{AF}_{1}$ and $\mathrm{AF}_{2}$ ), because we are dealing with two bands, the phase transition $\mathrm{AF}_{1} \rightarrow \mathrm{AF}_{2}$ would necessarily imply that the two spins' gaps abruptly interchange their sizes. Eventually, as pointed out earlier, a further variation of $W$ can cause the complete suppression of the IOAF ordering. The sequence of transitions $\mathrm{AF}_{1} \rightarrow \mathrm{AF}_{2} \rightarrow \mathrm{PM}$ should involve changes in the structure of the AF bands and, therefore, should be accompanied by Fermi surface (FS) reconstruction. One may also expect that other sequences of phase transition involving IOAF caused by increasing the field $h_{x}$ are also related to changes in the electronic structure.

We remark that the phase diagram temperature vs pressure of the IOAF, in the absence of a magnetic field, can be deduced from general arguments based on a Landau free energy with two order parameters. In the simplest situation, the two AF order parameters are linearly coupled [30] because both break time-reversal symmetry. In contrast, two order parameters that are even under time reversal couple quadratically lead to bicritical or tetracritical points [31]. In the first case, at very low temperatures, there should be a line of first-order transitions separating two distinct AF phases as an intensive thermodynamic parameter $g$ is varied. The line of first-order transitions ends at a critical end point (CEP) located at $\left(T_{\mathrm{CEP}}, g_{\mathrm{CEP}}\right)$. This kind of scenario was proposed by Mineev and Zhitomirsky (see Ref. [32]) to account for the temperature-pressure phase diagram of $\mathrm{URu}_{2} \mathrm{Si}_{2}$ [4]. In their description, the experimentally determined phase transition between the hidden ordered and the antiferromagnetic phases involves two types of antiferromagnetism: itinerant antiferromagnetism with small magnetic moments and localized antiferromagnetism with large magnetic moments. Unfortunately, there is strong evidence that the hidden order is not explainable in terms of conventional antiferromagnetic phases.

This paper is organized as follows. The UALM is presented in Sec. II. In Sec. III, we derive the Green's functions and the free energy. Next, in Sec. IV, we obtain the order parameters and gaps for zero and nonzero values of the transverse field $h_{x}$. A discussion of the numerical results follows in Sec. VII. The conclusions and other remarks are found in Sec. VIII.

\section{MODEL}

We will investigate a generic form of the UALM, which is written as

$$
\hat{H}=\hat{H}_{f}+\hat{H}_{d}+\hat{H}_{f d} .
$$

The $f$ electron part of Hamiltonian $\hat{H}_{f}$ is given by $\hat{H}_{f}=$ $\hat{H}_{f, 0}+\hat{H}_{f, \text { int }}$, where the noninteracting part $H_{0, f}$ describes two degenerate narrow $f$ bands and is expressed as

$$
\hat{H}_{f, 0}=\sum_{\underline{k}, \sigma} \sum_{\chi} E_{f}^{\chi}(\underline{k}) f_{\underline{k}, \sigma}^{\dagger \chi} f_{\underline{k}, \sigma}^{\chi} .
$$

The $\chi$ bands ( $\chi=\alpha$ and $\beta$ ) in Eq. (2) obey the intraband and interband nesting condition $E_{f}^{\chi}(\underline{k}+\underline{Q})=-E_{f}^{\chi^{\prime}}(\underline{k})$, where $\chi=\chi^{\prime}$ (intraband) or $\chi \neq \chi^{\prime}$ (interband). The vector $Q$ is a commensurate momentum transfer in the Brillouin zone. The 
interaction between the $f$ electrons is described by

$$
\begin{aligned}
& \hat{H}_{f, \text { int }}=\left(\frac{U-J}{2 N}\right) \sum_{\underline{k}, \underline{\underline{k}^{\prime}}, \underline{q}, \sigma, \chi \neq \chi^{\prime}} f_{\underline{k}+\underline{q}, \sigma}^{\dagger, \chi} f_{\underline{k}, \sigma}^{\chi} f_{\underline{k^{\prime}}-\underline{q}, \sigma}^{\dagger, \chi^{\prime}} f_{\underline{k}^{\prime}, \sigma}^{\chi^{\prime}} \\
& +\left(\frac{U}{2 N}\right) \sum_{\underline{k}, \underline{k^{\prime}}, \underline{q}, \sigma, \chi, \chi^{\prime}} f_{\underline{k}+\underline{q}, \sigma}^{\dagger, \chi} f_{\underline{k}, \sigma}^{\chi} f_{\underline{k^{\prime}}-\underline{q},-\sigma}^{\dagger, \chi^{\prime}} f_{\underline{k^{\prime}},-\sigma}^{\chi^{\prime}} \\
& +\left(\frac{J}{2 N}\right) \sum_{\underline{k}, \underline{k^{\prime}}, \underline{q}, \sigma, \chi \neq \chi^{\prime}} f_{\underline{k}+\underline{q}, \sigma}^{\dagger, \chi} f_{\underline{k}, \sigma}^{\chi^{\prime}} f_{\underline{k^{\prime}}-\underline{q},-\sigma}^{\dagger, \chi^{\prime}} f_{\underline{k}^{\prime},-\sigma}^{\chi},
\end{aligned}
$$

where $U$ is the screened Coulomb interaction and $J$ is the Hund's rule exchange. The conduction electron Hamiltonian $\hat{H}_{d}$ is expressed as

$$
\hat{H}_{d}=\sum_{\underline{k}, \sigma} \epsilon_{d}(\underline{k}) d_{\underline{k}, \sigma}^{\dagger} d_{\underline{k}, \sigma}
$$

where $\epsilon(\underline{k})$ describes the dispersion relation of conduction electrons labeled by the Bloch wave vector $\underline{k}$. The last term in Eq. (1) describes the on-site hybridization process in the UALM model by

$$
\hat{H}_{f d}=\sum_{\underline{k}, \sigma} \sum_{\chi=\alpha \beta}\left(V_{\chi}(\underline{k}) f_{\underline{k}, \sigma}^{\dagger, \chi} d_{\underline{k}, \sigma}+V_{\chi}^{*}(\underline{k}) d_{k, \sigma}^{\dagger} f_{\underline{k}, \sigma}^{\chi}\right) .
$$

In the present work, both the $5 f$ band $E_{f}^{\chi}(\underline{k})=\tilde{\epsilon}_{f}+\epsilon_{f}(\underline{k})$ and the conduction one $\epsilon_{d}(\underline{k})$ refer to a cubic lattice while $\tilde{\epsilon}_{f}$ is the band center. Thus

$$
\epsilon_{b}(\underline{k})=-2 t_{b}\left[\cos \left(k_{x} a\right)+\cos \left(k_{y} a\right)+\cos \left(k_{z} a\right)\right]
$$

in which $b=f$ or $d$ and $a$ is the lattice parameter.

The simplest possibility of a IOAF ordering with Ising anisotropy in a cubic lattice can be introduced by assuming that the lattice is bipartite. Therefore, we consider that

$$
n_{f, \underline{q}, \sigma}^{\chi}=\frac{n_{f}^{\chi}}{2} \delta_{\underline{q}, 0}+m_{f}^{\chi} \eta(\sigma) \delta_{\underline{q}, \pm \underline{Q}},
$$

where $n_{f}^{\chi}=n_{f, \uparrow}^{\chi}+n_{f, \downarrow}^{\chi}\left(n_{f}^{\chi}\right.$ is the $f$-electron average occupation of the $\chi$ band), $\eta(\uparrow)=+1$ or $\eta(\downarrow)=-1$, and $Q=$ $(\pi / a, \pi / a, \pi / a)$ is a commensurate nesting vector. Therefore, the modulation of the expectation value of the $z$ component $f$-electron spin density operator in real space for each orbital is $\left\langle\hat{S}_{z, \underline{r}_{j}}^{\chi}\right\rangle=m_{f}^{\chi} e^{i \underline{Q} \underline{\underline{r}}_{j}}$. The IOAF order parameters (i.e., the staggered magnetizations $m_{f}^{\alpha}$ and $m_{f}^{\beta}$ ) are obtained from

$$
m_{f}^{\chi}=\frac{1}{2}\left(n_{f, \underline{Q}, \uparrow}^{\chi}-n_{f, \underline{Q}, \downarrow}^{\chi}\right) .
$$

\section{GENERAL FORMULATION}

We include an applied magnetic field oriented transverse to the $z$ axis, which introduces an additional term into the Hamiltonian $\hat{H}_{\mathrm{ext}}=\hat{H}_{\mathrm{ext}}^{f}+\hat{H}_{\mathrm{ext}}^{d}$, where

$$
\hat{H}_{\mathrm{ext}}^{f}=-\Gamma_{f} \sum_{\underline{k}}\left(f_{\underline{k}, \uparrow}^{\dagger} f_{\underline{k}, \downarrow}+f_{\underline{k}, \downarrow}^{\dagger} f_{\underline{k}, \uparrow}\right),
$$

with

$$
\Gamma_{f}=g_{f} \mu_{B} h_{x}
$$

The term $\hat{H}_{\text {ext }}^{d}$ is the same as Eq. (9), except that the $f$ operators and the gyromagnetic factor $g_{f}$ are replaced by $d$ operators and $g_{d}$, respectively.

The temporal and spatial Fourier transform of the singleelectron $f$ - $f$ Green's function, within the Hartree-Fock approximation, satisfy the equations of motion given by

$$
\begin{aligned}
{[\omega-} & \left.\tilde{E}_{f}^{\alpha}(\underline{k})\right] G_{f f, \sigma}^{\alpha, \chi^{\prime}}\left(\underline{k}, \underline{k}^{\prime}, \omega\right) \\
= & \delta^{\alpha, \chi^{\prime}} \delta_{\underline{k}, \underline{k}^{k^{\prime}}} \delta_{\sigma, \sigma^{\prime}}+V_{\alpha}(\underline{k}) G_{d f, \sigma \sigma^{\prime}}^{\chi^{\prime}}\left(\underline{k}, \underline{k}^{\prime}, \omega\right) \\
& \quad-\Gamma_{f} G_{f f,-\sigma, \sigma}^{\beta, \chi^{\prime}}\left(\underline{k}, \underline{k}^{\prime}, \omega\right)+\phi_{\sigma}^{\alpha} G_{f f, \sigma, \sigma^{\prime}}^{\alpha, \chi^{\prime}}\left(\underline{k}+\underline{Q}, \underline{k}^{\prime}, \omega\right)
\end{aligned}
$$

and

$$
\begin{aligned}
{[\omega-} & \left.\tilde{E}_{f}^{\beta}(\underline{k})\right] G_{f f, \sigma, \sigma^{\prime}}^{\beta, \chi^{\prime}}\left(\underline{k}, \underline{k}^{\prime}, \omega\right) \\
= & \delta^{\beta, \chi^{\prime}} \delta_{\underline{k}, \underline{k}^{\prime}} \delta_{\sigma, \sigma^{\prime}}+V_{\beta}(\underline{k}) G_{d f, \sigma, \sigma^{\prime}}^{\chi^{\prime}}\left(\underline{k}, \underline{k}^{\prime}, \omega\right) \\
& \quad-\Gamma_{f} G_{f f,-\sigma \sigma^{\prime}}^{\alpha, \chi^{\prime}}\left(\underline{k}, \underline{k}^{\prime}, \omega\right)+\phi_{\sigma}^{\beta} G_{f f, \sigma, \sigma^{\prime}}^{\beta, \gamma^{\prime}}\left(\underline{k}+\underline{Q}, \underline{k}^{\prime}, \omega\right) .
\end{aligned}
$$

The spin-independent Hartree-Fock dispersion relation $\tilde{E}_{f}^{\chi}(\underline{k})$ is given by

$$
\tilde{E}_{f}^{\chi}(\underline{k})=E_{f}^{\chi}(\underline{k})+\sum_{\chi^{\prime}}\left((U-J) \frac{n_{f}^{\chi^{\prime}}}{2}\left(1-\delta^{\chi, \chi^{\prime}}\right)+U \frac{n_{f}^{\chi^{\prime}}}{2}\right),
$$

where the real function $\phi_{\sigma}^{\chi}$ is given by

$$
\phi_{\sigma}^{\chi}=\sum_{\chi^{\prime}}\left(U m^{\chi^{\prime}} \eta(-\sigma)+(U-J) m^{\chi}\left(1-\delta^{\chi, \chi^{\prime}}\right) \eta(\sigma)\right) .
$$

The mixed $f$ - $d$ Green's function satisfies the following equation:

$$
\begin{aligned}
{[\omega-} & \epsilon(\underline{k})] G_{d f, \sigma, \sigma^{\prime}}^{\chi^{\prime}}\left(\underline{k}, \underline{k}^{\prime}, \omega\right) \\
= & V_{\alpha}(\underline{k})^{*} G_{f f, \sigma, \sigma^{\prime}}^{\alpha, \gamma^{\prime}}\left(\underline{k}, \underline{k}^{\prime}, \omega\right)+V_{\beta}(\underline{k})^{*} G_{f f, \sigma, \sigma^{\prime}}^{\beta, \gamma^{\prime}}\left(\underline{k}, \underline{k}^{\prime}, \omega\right) \\
& \quad-\Gamma_{d} G_{d f,-\sigma, \sigma^{\prime}}^{\chi^{\prime}}\left(\underline{k}, \underline{k}^{\prime}, \omega\right) .
\end{aligned}
$$

We will choose a basis set for the $f$ orbitals, such that $V_{\beta}(\underline{k})=$ 0 and $V_{\alpha}(\underline{k})=V_{\alpha}$, simply to avoid the transformation to a new basis set. The choice of basis states should not change the main physical results, as discussed in Ref. [18]. The Green's function equations of motion given in Eqs. (11)-(15) form a closed set of equations, which can be solved exactly. The equations can be expressed in the matrix form

$$
\underline{\underline{\Pi}}^{\chi}(\underline{k}, \omega) \underline{\underline{G}}^{\chi \chi^{\prime}}\left(\underline{k}, \underline{k}^{\prime}, \omega\right)=\underline{\underline{\delta}}^{\chi^{\prime}}\left(\underline{k}, \underline{k}^{\prime}\right),
$$


where

$$
\begin{aligned}
& \underline{\underline{G}}^{\chi \chi^{\prime}}\left(\underline{k}, \underline{k^{\prime}}, \omega\right)=\left(\begin{array}{c}
G_{f f, \sigma \sigma^{\prime}}^{\chi \chi^{\prime}}\left(\underline{k}, \underline{k^{\prime}}, \omega\right) \\
G_{f f, \sigma \sigma^{\prime}}^{\chi x^{\prime}}\left(\underline{k}+\underline{Q}, \underline{k}^{\prime}, \omega\right) \\
G_{f f,-\sigma \sigma^{\prime}}^{\chi \chi^{\prime}}\left(\underline{k}, \underline{k}^{\prime}, \omega\right) \\
G_{f f,-\sigma \sigma^{\prime}}^{\chi \chi^{\prime}}\left(\underline{k}+\underline{Q}, \underline{k^{\prime}}, \omega\right)
\end{array}\right) \\
& \underline{\underline{\Pi}}^{\chi}(\underline{k}, \omega)=\left(\begin{array}{cccc}
\omega-\tilde{E}_{f}^{\chi}(\underline{k})-\xi_{\Gamma}^{\prime \chi}(\underline{k}) & -\phi_{\sigma}^{\chi} & \gamma_{\Gamma}^{\prime \chi}(\underline{k}) & 0 \\
-\phi_{\sigma}^{\chi} & \omega-\tilde{E}_{f}^{\chi}(\underline{k}+\underline{Q})-\xi_{\Gamma}^{\prime}(\underline{k}+\underline{Q}) & 0 & \gamma_{\Gamma}^{\prime \chi}(\underline{k}+\underline{Q}) \\
\gamma_{\Gamma}^{\prime \chi}(\underline{k}) & 0 & \omega-\tilde{E}_{f}^{\chi}(\underline{k})-\xi_{\Gamma}^{\prime \chi}(\underline{k}) & -\phi_{-\sigma}^{\chi} \\
0 & \gamma_{\Gamma}^{\prime \chi}(\underline{k}+\underline{Q}) & -\phi_{-\sigma}^{\chi} & \omega-\tilde{E}_{f}^{\chi}(\underline{k}+\underline{Q})-\xi_{\Gamma}^{\prime \chi}(\underline{k}+\underline{Q})
\end{array}\right), \\
& \underline{\underline{\delta}}^{\chi^{\prime}}\left(\underline{k}, \underline{k^{\prime}}\right)=\left(\begin{array}{c}
\delta^{x x^{\prime}} \delta_{\underline{k}, \underline{k}^{\prime}} \delta_{\sigma \sigma^{\prime}} \\
\delta^{x x^{\prime}} \delta_{\underline{k}+\underline{Q}, \underline{k}^{\prime}} \delta_{\sigma \sigma^{\prime}} \\
\delta^{x x^{\prime}} \delta_{\underline{k}, \underline{k}^{\prime}} \delta_{-\sigma \sigma^{\prime}} \\
\delta^{x x^{\prime}} \delta_{\underline{k}+\underline{Q}, \underline{k}^{\prime}} \delta_{-\sigma \sigma^{\prime}}
\end{array}\right) \text {, }
\end{aligned}
$$

with $\xi_{\Gamma}^{\prime \chi}(\underline{k})=\xi_{\Gamma}^{\chi}(\underline{k})\left(\delta_{\chi \alpha}+\left(1-\delta_{\chi \beta}\right)\right), \gamma_{\Gamma}^{\prime \chi}(\underline{k})=\gamma_{\Gamma}^{\chi}(\underline{k})\left(\delta_{\chi \alpha}\right.$ $\left.+\left(1-\delta_{\chi \beta}\right)\right)$ where

$$
\xi_{\Gamma}^{\chi}(\underline{k})=\frac{\left(\omega-\epsilon_{d}(\underline{k})\right)\left|V_{\chi}\right|^{2}}{\left(\omega-\epsilon_{d}(\underline{k})\right)^{2}-\Gamma_{d}^{2}}
$$

and

$$
\gamma_{\Gamma}^{\chi}(\underline{k})=\Gamma_{f}-\frac{\Gamma_{d}\left|V_{\chi}\right|^{2}}{\left(\omega-\epsilon_{d}(\underline{k})\right)^{2}-\Gamma_{d}^{2}}
$$

\section{INTRAORBITAL ANTIFERROMAGNETIC (IOAF) PHASE}

From now on, we will focus on the IOAF phases and their associated phase transitions.

\section{A. Order parameters and gaps with $\boldsymbol{h}_{x}=\mathbf{0}$}

The IOAF order parameters follow directly from the correlation functions $n_{f, Q, \sigma}^{\chi}$ [see Eq. (8)], which can be expressed as

$$
n_{f, \underline{Q}, \sigma}^{\chi}=\frac{1}{N} \sum_{\underline{k}, \sigma} \oint \frac{d \omega}{2 \pi i} f(\omega) G_{f f, \sigma}^{\chi \chi}(\underline{k}, \underline{k}+\underline{Q}, \omega) .
$$

The contour of the path integral encircles the real axis without enclosing any poles of the Fermi-Dirac distribution.

The correlation function $n_{f, \underline{Q}, \sigma}^{\beta}$ is found from the Green's function given in Eq. (B1). Therefore, from Eq. (8), one can obtain

$$
m_{f}^{\beta}=\left(U m_{f}^{\beta}+J m_{f}^{\alpha}\right) \chi_{f}^{\beta \beta}(\underline{Q}, 0)
$$

where

$$
\chi_{f}^{\beta \beta}(\underline{Q}, 0)=\frac{1}{N} \sum_{\underline{k}} \frac{f\left(E^{-}(\underline{k})\right)-f\left(E^{+}(\underline{k})\right)}{E^{+}(\underline{k})-E^{-}(\underline{k})}
$$

and $f(\omega)$ is the Fermi function.
The staggered magnetization of the $\alpha$ bands can be derived in a similar manner to Eq. (20). The result is

$$
m_{f}^{\alpha}=\left(U m_{f}^{\alpha}+J m_{f}^{\beta}\right) \chi_{f}^{\alpha \alpha}(\underline{Q}, 0),
$$

where $\chi_{f}^{\alpha \alpha}(\underline{Q}, 0)$ is now given as

$$
\begin{aligned}
\chi_{f}^{\alpha \alpha}(\underline{Q}, 0)= & \frac{1}{2 N} \sum_{\underline{k}, \sigma} \oint \frac{d \omega}{2 \pi i} f(\omega) \\
& \times \frac{\left(\omega-\epsilon_{d}(\underline{k})\right)\left(\omega-\epsilon_{d}(\underline{k}+\underline{Q})\right)}{D^{\alpha}(\omega, \underline{k})},
\end{aligned}
$$

with $D^{\alpha}$ given in Eq. (B6). The spin-independent quasiparticle bands are given by the solutions of $D^{\alpha}(\underline{k}, \omega)=0$.

Alternatively, one can formulate the self-consistency equations in terms of the gaps $\Delta_{\alpha(\beta)}$, since

$$
\phi_{\uparrow \downarrow}^{\alpha(\beta)}=\mp \Delta_{\alpha(\beta)},
$$

in which

$$
\Delta_{\alpha(\beta)}=U m_{f}^{\alpha(\beta)}+J m_{f}^{\beta(\alpha)} .
$$

The Hund's rule interaction couples the gap of a given band to the staggered magnetization of the other band. Using Eqs. (20), (21), (22), and (23), one may write the coupled equations for the gaps $\Delta_{\alpha}$ and $\Delta_{\beta}$ as

$$
U \Delta_{\alpha(\beta)}-J \Delta_{\beta(\alpha)}=\left(U^{2}-J^{2}\right) \Delta_{\alpha(\beta)} \chi_{f}^{\alpha \alpha(\beta \beta)}(\underline{Q}, 0) .
$$

\section{B. Order parameters and gaps with $\boldsymbol{h}_{\boldsymbol{x}} \neq \mathbf{0}$}

For $h_{x} \neq 0$, the pole structure of the Green's functions is much more complex, and is shown in Appendix A. For finite fields, the Green's functions $G_{f f, \sigma}^{\beta \beta}(\underline{k}, \underline{k}+\underline{Q}, \omega)$ and $G_{f f, \sigma}^{\alpha \alpha}(\underline{k}, \underline{k}+\underline{Q}, \omega)$ shown in Eqs. (A2) and (A6) can be used to obtain the order parameters $m_{\alpha}, m_{\beta}$ and the gaps following the same steps outlined in Sec. IV A. We assume that the $d$-conduction electron band is uncorrelated and wider than the correlated $f$ bands. We note that the magnetic field on the $d$ electrons, $\Gamma_{d}$, affects the order parameters $m^{\alpha}$ and $m^{\beta}$, mainly through the effect of the hybridization $V_{\alpha}$, and is 
small compared to the $\alpha$ and $\beta$ bandwidths. Therefore, it is reasonable to disregard the effects of $\Gamma_{d}$ on $m^{\alpha}$ and $m^{\beta}$.

\section{FREE ENERGY}

In the Hartree-Fock approximation, the free energy can be expressed in terms of the gaps by

$$
\begin{aligned}
f_{\mathrm{HF}}= & \Omega(T, \mu)+\mu N_{\mathrm{tot}} \\
& +\frac{N}{U^{2}-J^{2}}\left[U\left(\Delta_{\alpha}^{2}+\Delta_{\beta}^{2}\right)-2 J \Delta_{\alpha} \Delta_{\beta}\right],
\end{aligned}
$$

where $\mu$ is the chemical potential, $N_{\text {tot }}=n_{f}^{\alpha}+n_{f}^{\beta}+n_{d}\left(n_{d}\right.$ is the average occupation of the conduction electrons), and

$$
\Omega(T, \mu)=-k_{B} T \frac{1}{N} \sum_{\underline{k}} \sum_{\gamma} \ln \left[1+e^{-\frac{\left(E^{\gamma}-\mu\right)}{k_{B} T}}\right],
$$

where $N$ is the number of sites in the lattice. The quasiparticle energies $E^{\gamma}$ are obtained from the following: (i) for $h_{x}=0$, from Eq. (B2) and the roots of $D^{\alpha}(\omega, \underline{k})=0$ [see Eq. (B6)]; (ii) for $h_{x} \neq 0$, from Appendix $\mathrm{A}$, as roots of $\left|A^{\beta}\right|=0$ [see Eq. (A4)] and $\left|A^{\alpha}\right|=0$ [see Eq. (A9)] within the approximations $\gamma_{\Gamma}^{\chi}(\underline{k}) \approx \Gamma_{f}$ and $\xi_{\Gamma}^{\chi}(\underline{k}) \approx \frac{\left|V_{\chi}\right|^{2}}{\left(\omega-\epsilon_{d}(k)\right)}$, where we note that $\Gamma_{d} \approx 0$. It should be remarked that both $\left|A^{\alpha(\beta)}\right|$ depend directly on $\Delta_{\alpha(\beta)}^{2}$ [see Eq. (24)].

\section{INSTABILITY OF THE PARAMAGNETIC METALLIC (PM) PHASE}

In the general case $\left(h_{x}=0\right.$ and $\left.h_{x} \neq 0\right)$, a second-order instability of the PM phase can be determined from the linearized equations for the order parameters. The instability occurs when

$$
\begin{aligned}
(1 & \left.-U \chi_{f}^{\alpha \alpha(0)}(\underline{Q}, 0)\right)\left(1-U \chi_{f}^{\beta \beta(0)}(\underline{Q}, 0)\right) \\
& =J^{2} \chi_{f}^{\alpha \alpha(0)}(\underline{Q}, 0) \chi_{f}^{\beta \beta(0)}(\underline{Q}, 0) .
\end{aligned}
$$

This equation determines the Néel temperature $T_{N}$.

\section{RESULTS}

The numerical calculations were performed using a $\underline{k}$ independent hybridization $V_{\alpha}(\underline{k})=V_{\alpha}$ and a total occupancy of $N_{\text {tot }}=1.609$. This value of the total number of electrons was chosen such that the $5 f$ bands are close to half-filling, so that the instability of the paramagnetic state preferentially produces Néel antiferromagnetism. For wide conduction bands, due to an approximate electron-hole symmetry about $f$ occupancies at $n_{f}=2$ [33], similar phase diagrams are expected for $N_{\text {tot }}>2$. The parameters were chosen simply to highlight the existence of competing AF phases and their multicritical points and are not intended to be representative of any particular compound. The tight-binding parameters that we have used are $\tilde{\epsilon}_{f}=0.3 \mathrm{eV}, t_{d}=W_{d} / 6 \mathrm{eV}, t_{f}=W_{d} / 20 \mathrm{eV}$, $V_{\alpha}=1 / 10 \mathrm{eV}$, and $W_{f} / W_{d}=0.3$, where $2 W_{d(f)}$ is the width of the conduction $(5 f)$ band. From here on, we adopt the notation $W_{d}=W$. We also assume that the bandwidths $W_{d(f)}$ are sensitive to external pressure.

Phase diagrams are constructed from the self-consistent solutions of Eqs. (20) and (22) for the order parameters $m^{x}$. The

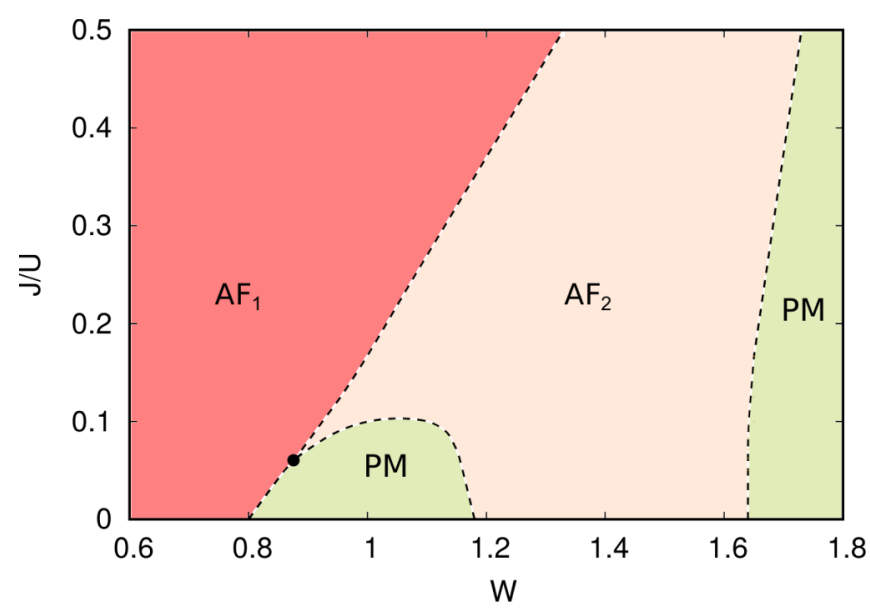

FIG. 1. Phase diagram for $J / U$ versus the bandwidth $W$ and $T=0$. All the phases transition are first order.

effect of Hund's rule exchange interaction strength $J$ on the boundaries of the phases $\mathrm{AF}_{1}, \mathrm{AF}_{2}$, and $\mathrm{PM}$, as $W$ increases, is shown in Fig. 1, for $T=0$. The phase $\mathrm{AF}_{1}$ is characterized by $m^{\beta}>m^{\alpha}$, while the $\mathrm{AF}_{2}$ denotes the phase where $m^{\alpha}>m^{\beta}$. The first-order line $\mathrm{AF}_{1} \rightarrow \mathrm{AF}_{2}$ ends at a quantum triple point located at $(J / U)_{\text {tri }} \approx 0.07$ and $W_{\text {tri }} \approx 0.85$, where the $\mathrm{AF}_{1}, \mathrm{AF}_{2}$, and $\mathrm{PM}$ phases coexist. We shall now remark on the effect that the Hund's rule exchange has on the phase diagram. For $J=0, m_{\beta}$ and $m_{\alpha}$ are completely independent [see Eqs. (20) and (22)]. In this case, the condition for opening of gaps is satisfied for both bands in the region of $W \lesssim 0.8$, but for $W \gtrsim 1.1$ only the $\alpha$ band satisfies such a condition. That is, in the $\mathrm{AF}_{2}$ phase the order parameter only has $\alpha$ character, i.e., $m_{\alpha}>m_{\beta}=0$. In this case, the transitions are $\mathrm{AF}_{1} \rightarrow \mathrm{PM}$ and $\mathrm{PM} \rightarrow \mathrm{AF}_{2}$, as the bandwidth $W$ is increased. For $J$ finite but small, $m_{\alpha}$ and $m_{\beta}$ are both finite in the $\mathrm{AF}_{2}$ phase. However, the conditions for opening the gap for both bands are still not satisfied within the intermediate interval of $W$. As $J$ further increases, the coupling between the two order parameters also increases. Above a certain value of $J$, the condition for opening of gaps for both $\alpha$ and $\beta$ bands are fully recovered. This shows that there is a threshold of $J$, above which the direct transition $\mathrm{AF}_{1} \rightarrow \mathrm{AF}_{2}$ occurs. The region above the threshold is the focus of the present investigation. Therefore, from now on, we use $J=U / 5$ with $U=0.165 \mathrm{eV}$.

The staggered magnetizations $m^{\alpha}$ and $m^{\beta}$ at finite temperatures are shown in Figs. 2(a) and 2(b), respectively. For $k_{B} T=$ 0 , both order parameters exhibit two discontinuities, one at $W \approx 1.05$ and another at $W \approx 1.65$. These discontinuities indicate the occurrence of first-order phase transitions. The first transition, occurring at $W \approx 1.05$, is between two types of antiferromagnetic phases. It should be remarked that $m_{\alpha} \lessgtr$ $m_{\beta}$ implies that $\Delta_{\alpha} \lessgtr \Delta_{\beta}$. There is another phase transition $\mathrm{AF}_{2} \rightarrow$ PM. The inset in Fig. 2(a) exhibits, in detail, the region of $W$ and $T$ where this last transition takes place.

In Fig. 3, the dependence of the $T=0$ energy per unit cell $E_{N}$ on the possible values of the gaps is shown for particular values of $W$. The ground state is determined by the global minimum. This figure illustrates the competition between the $\operatorname{AF}_{1}\left(\Delta_{\beta}>\Delta_{\alpha}\right), \mathrm{AF}_{2}\left(\Delta_{\beta}<\Delta_{\alpha}\right)$, and PM phases. The 

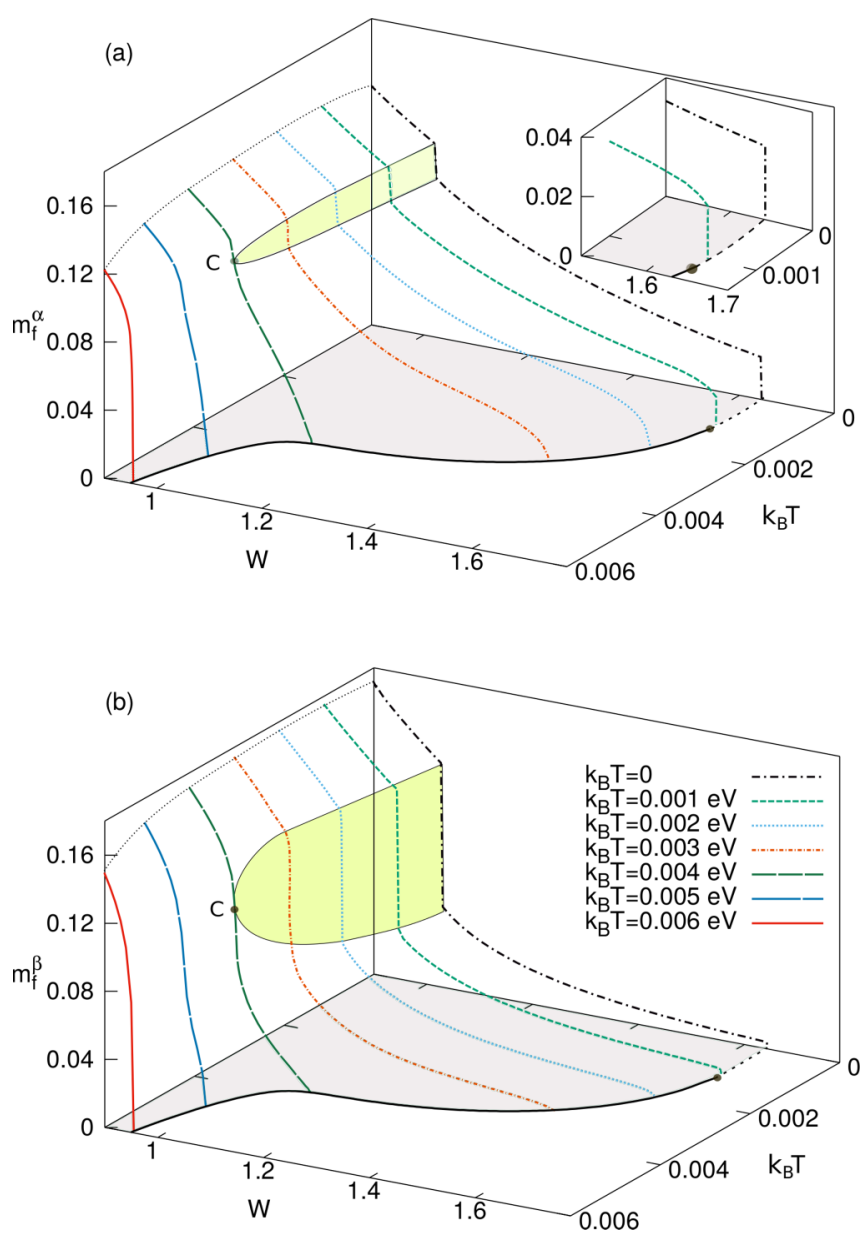

FIG. 2. (a) Dependence of the order parameter $m_{\alpha}$ on the bandwidth $W$ and temperature $T$. (b) The same for the order parameter $m_{\beta}$.

energies $E_{N}$ are projected onto the $\Delta_{\alpha} \times \Delta_{\beta}$ plane, so the darkest regions serve as indicators for the values of $\Delta_{\alpha}$ and $\Delta_{\beta}$ that correspond to the ground states. Figures 3(a) and 3 (b) show the interchange of global minimum between $\mathrm{AF}_{1}$ and $\mathrm{AF}_{2}$ phases. In Figs. 3(c) and 3(d), $E_{N}$ is shown for two different values of $W$, which confirms the existence of a new first-order transition $\mathrm{AF}_{2} \rightarrow \mathrm{PM}$. For $W=1.645$, the global minimum of the energy $E_{N}$ corresponds to the nontrivial solution, while for $W=1.67$ the global minimum is found for the trivial solution.

Based on Eq. (29), the behavior of the order parameters $m^{\alpha}$ and $m^{\beta}$, and the free energy given in terms of gaps $\Delta_{\alpha}$ and $\Delta_{\beta}$, it is possible to construct the phase diagram shown in Fig. 4. First, there is a second-order transition at the Néel temperature $T_{N}$ (denoted by a solid line), which is marked by the formation of the AF gaps. Second, for $0.95<W<1.05$, there is a direct first-order transition $\mathrm{AF}_{1} \rightarrow \mathrm{AF}_{2}$, which ends at a CEP located at $k_{B} T_{\mathrm{CEP}} \approx 0.0038$ and $W_{\mathrm{CEP}} \approx 0.9826$. Our analysis shows that the Hund's rule exchange interaction produces a term in the free energy that is bilinear in $m_{\alpha}$ and $m_{\beta}$. From Eqs. (27) and (28), an expansion of the free energy occurs in terms of even powers of the gaps which are linear combinations of both $m^{\alpha}$ and $m^{\beta}$ [see Eq. (25)]. We remark that, in the range $T_{\mathrm{CEP}}<T<T_{N}$, the jump in the order parameters
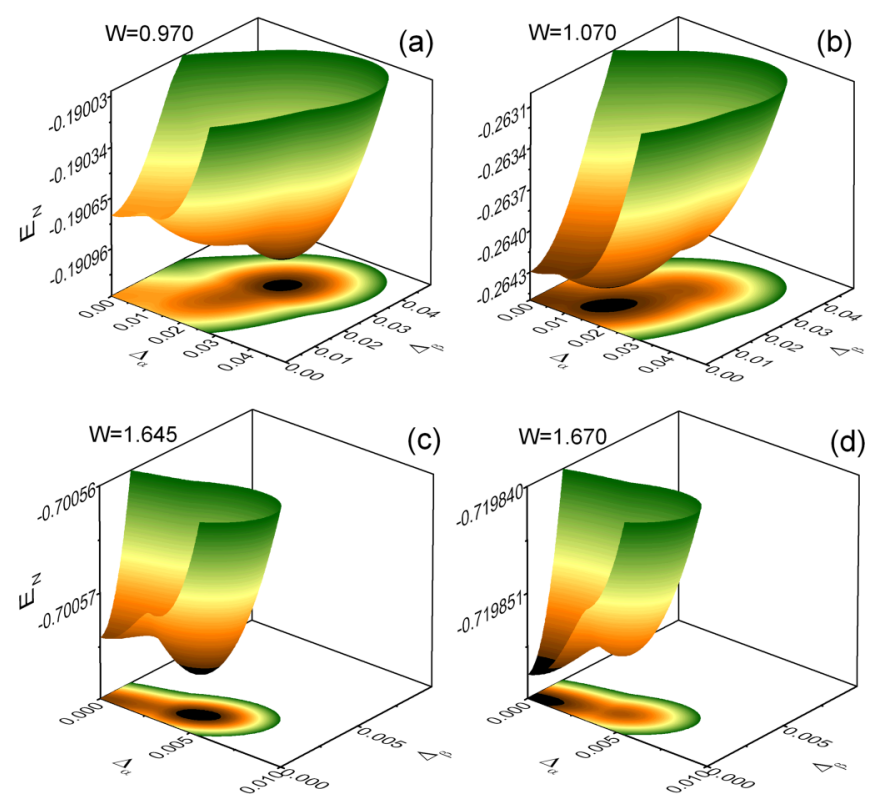

FIG. 3. Zero temperature value of the energy per unit cell. The projection of the ground state energy on the plan of the gaps show dark regions, in which the self-consistent solutions for the gaps $\Delta_{\alpha}$ and $\Delta_{\beta}$ are to be found. The sequence of panels shows the competition between the two types of orders as the bandwidth $W$ is varied.

becomes smooth and the two AF phases can be continuously connected by a path that bypasses the CEP. The phase diagram is completed by a third line of transitions $\mathrm{AF}_{2} \rightarrow \mathrm{PM}$, which occurs for $1.6<W<1.7$. The line of transitions changes from a second-order to a first-order transition at a tricritical point (TCP) located at $k_{B} T_{\mathrm{TCP}} \approx 0.0011$ and $W_{\mathrm{TCP}} \approx 1.6410$.

The partial densities of states (DOS) shown in Fig. 5 are for bandwidths and temperatures in close proximity to the dashed line, which separates the phases $\mathrm{AF}_{1}$ and $\mathrm{AF}_{2}$ at $T=0$, and also at temperatures where the two AFs can be connected smoothly. For the $\beta f$ electron, there is an insulator $\rightarrow$ metal transition at which the $\alpha$ states maintain their metallic characters. The electronic structure transition is observed to

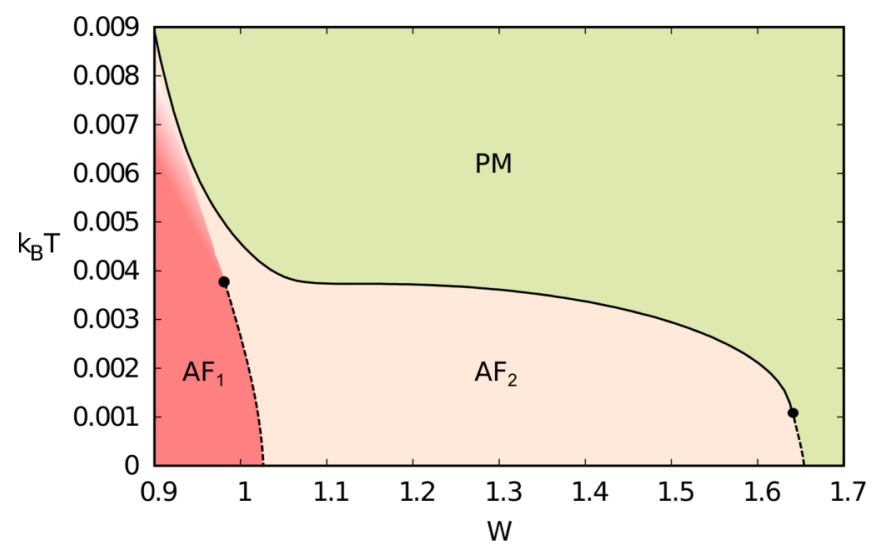

FIG. 4. Phase diagram for the temperature versus the bandwidth $W$. The solid and the dashed lines denote second-order and first-order transition, respectively. 

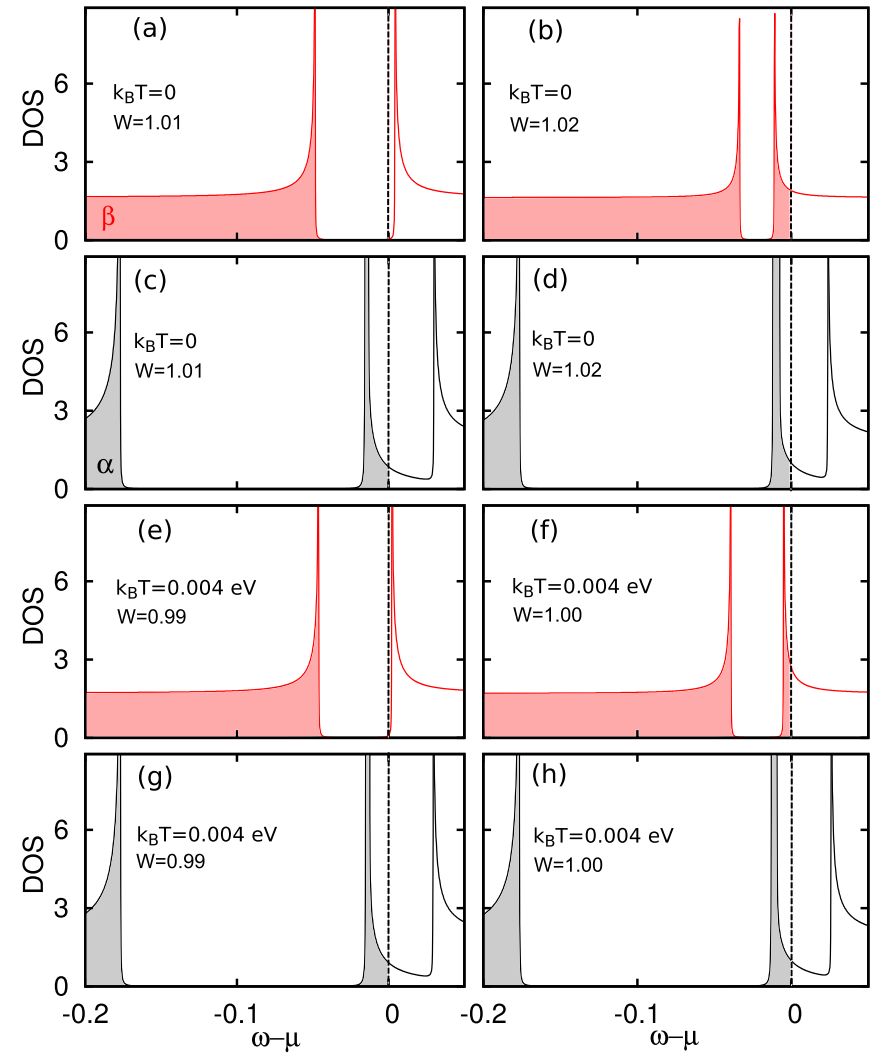

FIG. 5. Panels show the $\alpha$ and $\beta$ partial densities of states for values of $W$ closed to the dashed line $\left(\right.$ close $\left.\mathrm{AF}_{1}\right)$ of the phase diagram (see Fig. 4).

start at $T=0$, which exactly coincides with the line of the first-order transitions $\mathrm{AF}_{1} \rightarrow \mathrm{AF}_{2}$, persisting above the CEP.

The zero field magnetic phase diagram changes drastically when a transverse field $h_{x}$ is applied. The resulting $k_{B} T$ versus the bandwidth $W$ phase diagram is shown in Fig. 6 where the values of $\Gamma_{f}$ are directly proportional to $h_{x}$ [see Eq. (10)]. The main effect of $\Gamma_{f}$ is to separate the phases $\mathrm{AF}_{1}$ and $\mathrm{AF}_{2}$, creating a dome-shaped region for this phase with two TCPs. As $\Gamma_{f}$ increases, the $\mathrm{AF}_{2}$ domed-shaped region decreases until its complete suppression. We remark that $m^{\alpha}$ is less affected by $\Gamma_{f}$ in the region of $W \lesssim 1$ than in the region of $W \gtrsim 1$. For $\Gamma_{f}=0.035, m^{\alpha}$ is completely suppressed for $W \gtrsim 1$. In fact, the behavior of the order parameters $m^{\alpha}$ and $m^{\beta}$ are closely related to the condition for opening the gap for the $\alpha$ and $\beta$ bands. The magnetic field produces a $\underline{k}$-dependent shift, which depends on the spin $\sigma$. In addition, the Fermi energy $\left(E_{F}\right)$ is shifted to higher energies. Therefore, for sufficiently high values of $\Gamma_{f}$, the Fermi surface is no longer nested. Nevertheless, the electronic characters of the $\mathrm{AF}_{1}$ and $\mathrm{AF}_{2}$ phases are unchanged.

The evolution of the phase diagram of Fig. 6 can be better understood in terms of the condition for opening the gaps in the $\beta$ and $\alpha$ bands. For $\Gamma_{f}=0$, the $\beta$ sheet of the Fermi surface is nested when $E_{f}^{\beta}(\underline{k})=E_{f}^{\beta}(\underline{k}+\underline{Q})=\mu$. The presence of $h_{x}$ produces a $\underline{k}$-dependent spin splitting of the dispersion relation, which can result in a shift of $\mu$. The evolution of the gapped regions of $\beta$ and $\alpha$ bands with
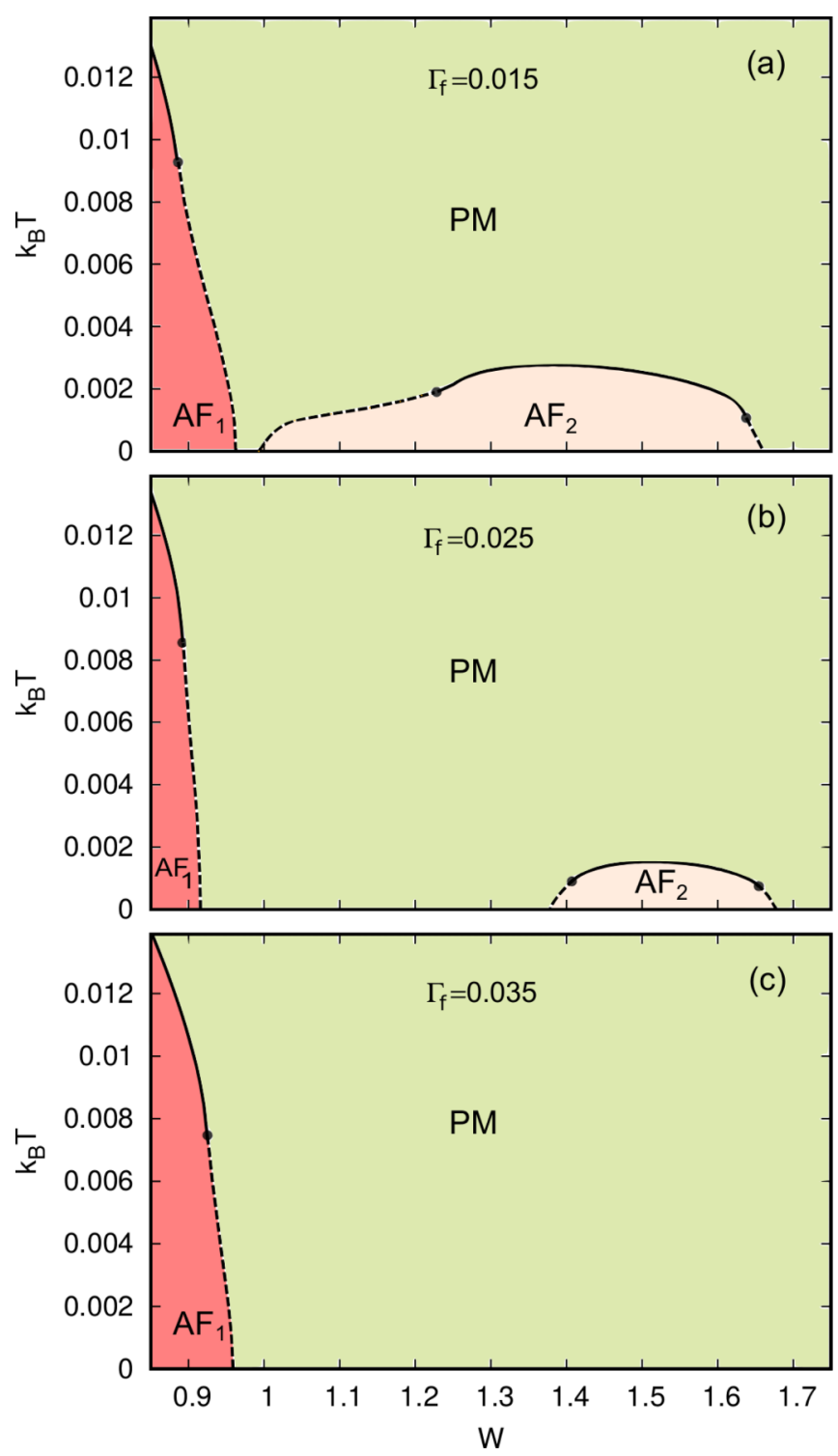

FIG. 6. Phase diagram of the temperature versus the bandwidth $W$ for different values of $\Gamma_{f}$. The solid lines denote second-order transitions while the dashed lines denote first-order transitions.

increasing $\Gamma_{f}$ is shown in Figs. 7 and 8. The $\alpha$ bands involve the hybridization $V_{\alpha}$, which also affects the band's dispersion relation. In Fig. 7, the dispersion relation is calculated for a bandwidth of $W=0.9$, which places the system in the $\mathrm{AF}_{1}$ phase (see Fig. 6). The Fermi energy $E_{F}\left(\mu=E_{F}\right)$ is positioned within the gap in the $\beta$-band dispersion relation for all values of $\Gamma_{f}$. Meanwhile, at the gapped region, the extent to which the $\alpha$-band dispersion relation dips below the Fermi energy decreases with increasing $\Gamma_{f}$. Consequently, the nesting of the $\alpha$ band is affected more strongly than the $\beta$ band. The results show that the AF phases are more stable than the paramagnetic phase, if the Fermi energy (or $\mu$ ) is inside of both, or either one or other of the $\alpha$ or $\beta$ gaps.

We find a different situation for $W=1.5\left(\mathrm{AF}_{2}\right.$ phase $)$, as shown in Fig. 8. The gap in the $\beta$ band is always below the Fermi energy, whereas the Fermi energy lies within the gap of the $\alpha$ band. However, as $\Gamma_{f}$ increases, the Fermi energy tends 


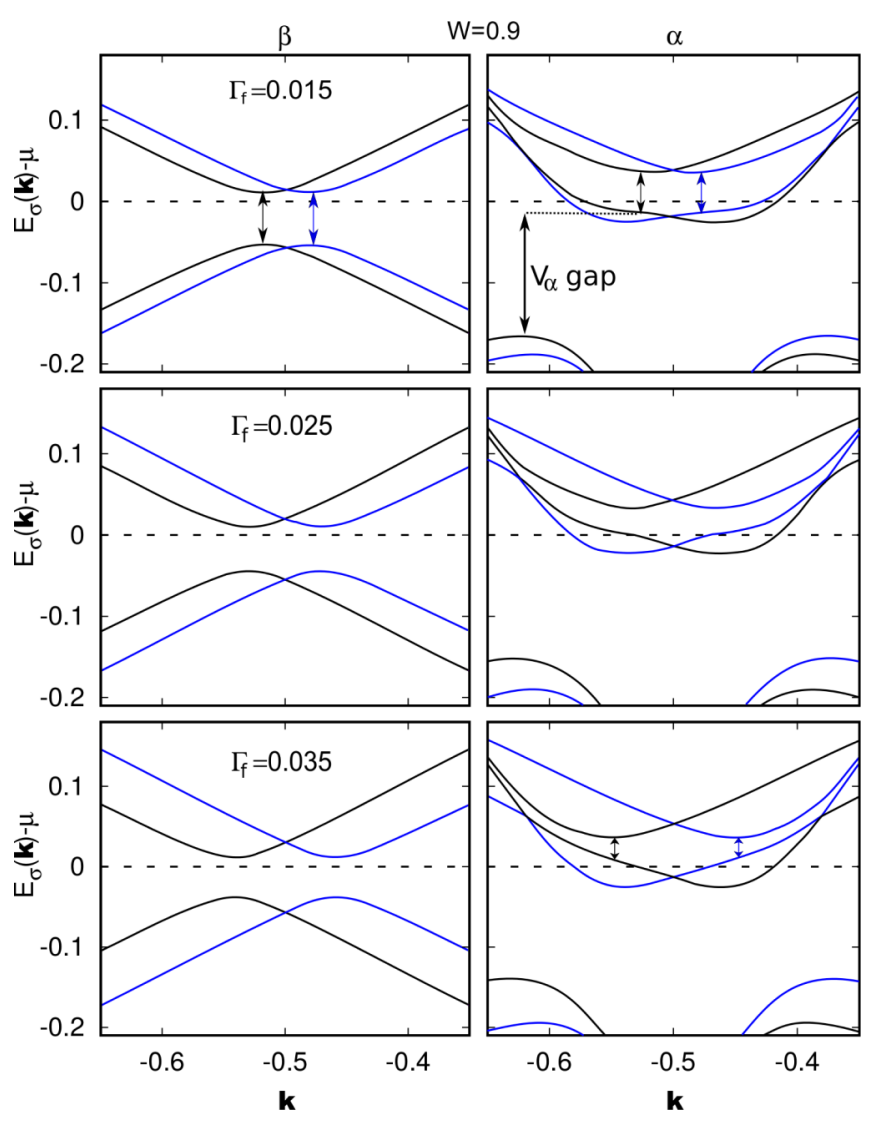

FIG. 7. Electronic dispersion relations near the gaps [along the direction $(\pi, \pi, \pi)-(0,0,0)]$ for $W=0.9, T=0$, and different values of $\Gamma_{f}$. The blue and black colors represent the up and down spin subbands, respectively. The double arrows indicate where the AF gaps occur.

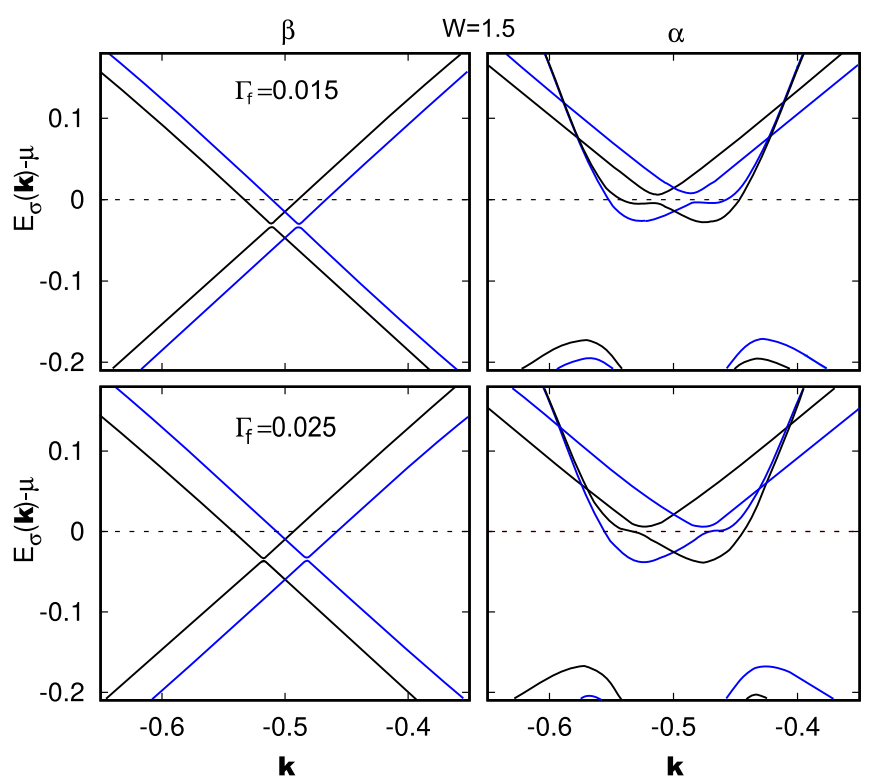

FIG. 8. Dispersion relations near the electronic gaps [along the direction $(\pi, \pi, \pi)-(0,0,0)]$ for $W=1.5, T=0$, and different values of $\Gamma_{f}$. The blue and black colors represent the bands with spin-up and spin-down subbands, respectively. to move to the bottom of the $\alpha$ gap, until for $\Gamma_{f}=0.035$ (not shown here), the Fermi energy falls below the gap as in the $\beta$ band case. When both gaps are below the Fermi energy, the bands are not nested and the paramagnetic phase is more stable.

\section{CONCLUSIONS}

In this work, we have investigated the unfolding of an itinerant antiferromagnetic phase and the subsequent emergence of multicritical points due to the competition between the unfolded phases that appear in a model suitable to describe uranium compounds. This model describes two narrow $5 f$ bands $(\alpha$ and $\beta$ ) that are hybridized asymmetrically with a single conduction band. Besides the direct Coulomb interaction between electrons in the same $5 f$ band, there is a Hund's rule exchange interaction between electrons in the different bands. We have obtained temperature vs pressure phase diagrams with and without the application of a magnetic field. We have assumed that pressure is associated with bandwidth variation. Moreover, given that the order parameter has an Ising-like anisotropy, the magnetic field is considered transversal to this anisotropy direction.

Therefore, we show that there is an unfolding of the antiferromagnetic phase into two distinct $\mathrm{AF}_{1}$ and $\mathrm{AF}_{2}$ phases. These phases are characterized by having finite staggered magnetization for both bands. Thus $\mathrm{AF}_{1}$ and $\mathrm{AF}_{2}$ are given by $m_{\beta}>m_{\alpha}$ and $m_{\alpha}>m_{\beta}$, respectively. The role of the Hund's rule exchange interaction is essential in producing these two types of phases because this interaction couples the staggered magnetization in different bands. Most important for the purpose of this work is that from this kind of phase competition emerges a particular set of multicritical points. In the absence of a magnetic field, there is a critical end point (CEP) and a tricritical point (TCP), respectively, in the phase transitions $\mathrm{AF}_{1} \rightarrow \mathrm{AF}_{2}$ and $\mathrm{AF}_{2} \rightarrow \mathrm{PM}$ as the bandwidth increases. The presence of the CEP in our phase diagram is in accordance with the description based on the generic two order parameter Landau free energy described in Ref. [32], where the order parameters were assumed to be odd under time reversal. For a finite magnetic transverse field $h_{x}$, the phase diagram is drastically changed. The direct transition between the AF phases is replaced by a reentrant sequence of transitions $\mathrm{AF}_{1} \rightarrow \mathrm{PM} \rightarrow \mathrm{AF}_{2} \rightarrow \mathrm{PM}$. The $\mathrm{AF}_{2}$ phase acquires a dome shape. Consequently, the initial set of multicritical points is also completely changed. For instance, the CEP is suppressed. The $\mathrm{AF}_{1}$ line transition has one TCP, while the dome shaped $\mathrm{AF}_{2}$ line transition has two TCPs. All TCPs are affected relatively weakly by further increases of $h_{x}$. In fact, the dome is gradually suppressed by the field until its complete disappearance, $\beta$ (related to the respective bands).

It should be remarked that the shape of the phase diagram with and without $h_{x}$ can be related to changes in the electronic structure. For $h_{x}=0$, as the bandwidth of the two $5 f$ bands increases, our results show that the $\beta f$ electrons undergo a insulator $\rightarrow$ metal transition, while the $\alpha$ electrons maintain their metallic characters. Thus our electronic structure can be described in terms of a transition between half to full metallicity. Below the CEP, this transition exactly coincides with the $\mathrm{AF}_{1} \rightarrow \mathrm{AF}_{2}$ first-order transition. The analysis of 
the electronic dispersion relation shows that the presence of a magnetic field $h_{x}$ does not change the metallic character of both $\mathrm{AF}_{1}$ and $\mathrm{AF}_{2}$ phases, at least for the range of $h_{x}$ considered in the present work.

To conclude, we highlight that our results show a detailed evolution of multicritical points when pressure and magnetic field are applied simultaneously. As far as we know, there are few theoretical results in the literature showing this particular evolution. Although our results refer to a specific model of two $5 f$ degenerate narrow bands, they can shed light on the growing field of the multicritical (classical and quantum) points in the physics of uranium compounds.

\section{ACKNOWLEDGMENTS}

The present study was supported by the Brazilian agencies Conselho Nacional de Desenvolvimento Científico e Tecnológico (CNPq), Coordenação de Aperfeiçoamento de Pessoal de Nível Superior (CAPES), and Fundação de Amparo à pesquisa do Estado do RS (FAPERGS).

\section{APPENDIX A}

In the case where $V_{\beta}=0$, by using Eq. (16), the Green's function can be explicitly written as

$$
\begin{gathered}
G_{f f, \sigma \sigma^{\prime}}^{\beta \chi^{\prime}}\left(\underline{k}, \underline{k^{\prime}}, \omega\right)=\delta^{\beta \chi^{\prime}} \delta_{\underline{k}, \underline{k}^{\prime}} \delta_{\sigma \sigma^{\prime}} \frac{\left[D_{0-\sigma}^{\beta}(\omega, \underline{k})\left(\omega-\tilde{E}_{f}^{\beta}(\underline{k}+\underline{Q})\right)-\Gamma_{f}^{2}\left(\omega-\tilde{E}_{f}^{\beta}(\underline{k})\right)\right]}{\left|A^{\beta}\right|}, \\
G_{f f, \sigma \sigma^{\prime}}^{\beta \chi^{\prime}}\left(\underline{k}+\underline{Q}, \underline{k^{\prime}}, \omega\right)=\delta^{\beta \chi^{\prime}} \delta_{\underline{k}+\underline{Q}, \underline{k^{\prime}}} \delta_{\sigma \sigma^{\prime}} \frac{\left[\Gamma_{f}^{2} \phi_{-\sigma}^{\beta}+\phi_{\sigma}^{\beta} D_{0-\sigma}^{\beta}(\omega, \underline{k})\right]}{\left|A^{\beta}\right|},
\end{gathered}
$$

where

$$
D_{0 \sigma}^{\beta}(\omega, \underline{k})=\left(\omega-\tilde{E}_{f}^{\beta}(\underline{k}+\underline{Q})\right)\left(\omega-\tilde{E}_{f}^{\beta}(\underline{k})\right)-\left(\phi_{\sigma}^{\beta}\right)^{2}
$$

and

$$
\left|A^{\beta}\right|=D_{0 \sigma}^{\beta}(\omega, \underline{k}) D_{0-\sigma}^{\beta}(\omega, \underline{k})+\Gamma_{f}^{2}\left[\Gamma_{f}^{2}-2 \phi_{\sigma}^{\beta} \phi_{-\sigma}^{\beta}\right]-\Gamma_{f}^{2}\left[\left(\omega-\tilde{E}_{f}^{\beta}(\underline{k})\right)^{2}+\left(\omega-\tilde{E}_{f}^{\beta}(\underline{k}+\underline{Q})\right)^{2}\right] .
$$

Moreover,

$$
\begin{gathered}
G_{f f, \sigma \sigma^{\prime}}^{\alpha \chi^{\prime}}\left(\underline{k}, \underline{k^{\prime}}, \omega\right)=\delta^{\alpha \chi^{\prime}} \delta_{\underline{k}, \underline{k}^{\prime}} \delta_{\sigma \sigma^{\prime}} \frac{(\omega-\epsilon(\underline{k}))}{\left|A^{\alpha}\right|} A_{1 \sigma}^{\alpha}(\omega, \underline{k}), \\
G_{f f, \sigma \sigma^{\prime}}^{\alpha \chi^{\prime}}\left(\underline{k}+\underline{Q}, \underline{k^{\prime}}, \omega\right)=\delta^{\alpha \chi^{\prime}} \delta_{\underline{k}+\underline{Q}, \underline{k}^{\prime}} \delta_{\sigma \sigma^{\prime}} A_{2 \sigma}^{\alpha}(\omega, \underline{k}) \frac{(\omega-\epsilon(\underline{k}))(\omega-\epsilon(\underline{k}+\underline{Q}))}{\left|A^{\alpha}\right|},
\end{gathered}
$$

where

$$
\begin{aligned}
A_{1 \sigma}^{\alpha}(\omega, \underline{k})= & D_{0}^{\alpha}(\omega, \underline{k})\left(D_{0}^{\alpha}(\omega, \underline{k}+\underline{Q})\right)^{2}-\left(\gamma_{\Gamma}^{\alpha \alpha}(\underline{k}+\underline{Q})\right)^{2} D_{0}^{\alpha}(\omega, \underline{k})(\omega-\epsilon(\underline{k}+\underline{Q}))^{2} \\
& -\left(\phi_{-\sigma}^{\alpha}\right)^{2} D_{0}^{\alpha}(\omega, \underline{k}+\underline{Q})(\omega-\epsilon(\underline{k}+\underline{Q}))(\omega-\varepsilon(\underline{k}))
\end{aligned}
$$

and

$$
\begin{aligned}
A_{2 \sigma}^{\alpha}(\omega, \underline{k})= & \phi_{\sigma}^{\alpha}\left[D_{0}^{\alpha}(\omega, \underline{k}) D_{0}^{\alpha}(\omega, \underline{k}+\underline{Q})-\left(\phi_{-\sigma}^{\alpha}\right)^{2}(\omega-\epsilon(\underline{k}))(\omega-\varepsilon(\underline{k}+\underline{Q}))\right] \\
& +\phi_{-\sigma}^{\alpha} \gamma_{\Gamma}^{\alpha \alpha}(\underline{k}) \gamma_{\Gamma}^{\alpha \alpha}(\underline{k}+\underline{Q})(\omega-\epsilon(\underline{k}))(\omega-\epsilon(\underline{k}+\underline{Q})),
\end{aligned}
$$

with

$$
\begin{aligned}
\left|A^{\alpha}\right|= & {\left[D_{0}^{\alpha}(\omega, \underline{k}) D_{0}^{\alpha}(\omega, \underline{k}+\underline{Q})-\left(\phi_{-\sigma}^{\alpha}\right)^{2}(\omega-\epsilon(\underline{k}+\underline{Q}))(\omega-\epsilon(\underline{k}))\right]\left[D_{0}^{\alpha}(\omega, \underline{k}) D_{0}^{\alpha}(\omega, \underline{k}+\underline{Q})-\left(\phi_{\sigma}^{\alpha}\right)^{2}\right.} \\
& \times(\omega-\epsilon(\underline{k}+\underline{Q}))(\omega-\epsilon(\underline{k}))]+\left[\left(\gamma_{\Gamma}^{\alpha \alpha}(\underline{k})\right)^{2}\left(\gamma_{\Gamma}^{\alpha \alpha}(\underline{k}+\underline{Q})\right)^{2}-2 \phi_{\sigma}^{\alpha} \phi_{-\sigma}^{\alpha} \gamma_{\Gamma}^{\alpha \alpha}(\underline{k}) \gamma_{\Gamma}^{\alpha \alpha}(\underline{k}+\underline{Q})\right](\omega-\epsilon(\underline{k}))^{2}(\omega-\epsilon(\underline{k}+\underline{Q}))^{2} \\
& -\left(\gamma_{\Gamma}^{\alpha \alpha}(\underline{k})\right)^{2}\left(D_{0}^{\alpha}(\omega, \underline{k}+\underline{Q})\right)^{2}(\omega-\epsilon(\underline{k}))^{2}-\left(\gamma_{\Gamma}^{\alpha \alpha}(\underline{k}+\underline{Q})\right)^{2}\left(D_{0}^{\alpha}(\omega, \underline{k})\right)^{2}(\omega-\epsilon(\underline{k}+\underline{Q}))^{2},
\end{aligned}
$$


where $D_{0}^{\alpha}(\omega, \underline{k}), \phi_{\sigma}^{\chi}$, and $\gamma_{\Gamma}^{\chi \chi}(\underline{k})$ are defined in Eqs. (B7), (14), and (18), respectively. From the equations $\left|A^{\beta}\right|=0$ and $\left|A^{\alpha}\right|=0$, the spin independent quasiparticle energies are $E^{\gamma}$, where $\gamma$ is the number of solutions. For $\gamma_{\Gamma}^{\chi}(\underline{k}) \approx \Gamma_{f}$ and $\xi_{\Gamma}^{\chi}(\underline{k}) \approx \frac{\left|V_{\chi}\right|^{2}}{\left(\omega-\epsilon_{d}(k)\right)}$, the equation $\left|A^{\beta}\right|=0$ has $\gamma=1 \ldots 4$, while $\left|A^{\alpha}\right|=0$ has $\gamma=1 \ldots 8$.

\section{APPENDIX B}

The Green's function $G_{f f, \sigma}^{\beta \beta}(\underline{k}, \underline{k}+\underline{Q}, \omega)$ with $h_{x}=0$ acquires a simple form given by

$$
G_{f f, \sigma}^{\beta \beta}(\underline{k}, \underline{k}+\underline{Q}, \omega)=\phi_{\sigma}^{\beta}\left(\frac{\left|\widetilde{B}^{+}(\underline{k})\right|^{2}}{\omega-E_{+}(\underline{k})}+\frac{\left|\widetilde{B}^{-}(\underline{k})\right|^{2}}{\omega-E_{-}(\underline{k})}\right),
$$

where the spin-independent quasiparticle bands $E_{ \pm}(\underline{k})$ are

$$
E_{ \pm}(\underline{k})=\left(\frac{\tilde{E}_{f}^{\beta}(\underline{k})+\tilde{E}_{f}^{\beta}(\underline{k}+\underline{Q})}{2}\right) \pm X_{\underline{k}}
$$

with

$$
X_{\underline{k}}=\sqrt{\frac{\tilde{E}_{f}^{\beta}(\underline{k})-\tilde{E}_{f}^{\beta}(\underline{k}+\underline{Q})}{2}+\left(U m_{f}^{\beta}+J m_{f}^{\alpha}\right)^{2}}
$$

and the spectral weights $\left|\widetilde{B}^{ \pm}(\underline{k})\right|^{2}$ in Eq. (B1) found as

$$
\left|\widetilde{B}^{ \pm}(\underline{k})\right|^{2}= \pm \frac{1}{2} \frac{1}{\sqrt{\frac{\tilde{E}_{f}^{\beta}(\underline{k})-\tilde{E}_{f}^{\beta}(\underline{k}+\underline{Q})}{2}+\left(U m_{f}^{\beta}+J m_{f}^{\alpha}\right)^{2}}} .
$$

Meanwhile, the $\alpha$ band has no simple form for the Green's function $G_{f f, \sigma}^{\alpha \alpha}(\underline{k}, \underline{k}+\underline{Q}, \omega)$,

$$
G_{f f, \sigma}^{\alpha \alpha}(\underline{k}, \underline{k}+\underline{Q}, \omega)=\phi_{\sigma}^{\alpha} \frac{\left(\omega-\epsilon_{d}(\underline{k})\right)\left(\omega-\epsilon_{d}(\underline{k}+\underline{Q})\right)}{D^{\alpha}(\omega, \underline{k})},
$$

with

$$
\begin{aligned}
D^{\alpha}(\underline{k}, \omega)= & D_{0}^{\alpha}(\omega, \underline{k}) D_{0}^{\alpha}(\omega, \underline{k}+\underline{Q}) \\
& -\left(U m_{f}^{\alpha}+J m_{f}^{\beta}\right)^{2}\left(\omega-\epsilon_{d}(\underline{k})\right)\left(\omega-\epsilon_{d}(\underline{k}+\underline{Q})\right)
\end{aligned}
$$

and

$$
D_{0}^{\alpha}(\underline{k}, \omega)=\left(\omega-\tilde{E}_{f}^{\alpha}(\underline{k})\right)\left(\omega-\epsilon_{d}(\underline{k})\right)-\left|V_{\alpha}\right|^{2} .
$$

[1] K. T. Moore and G. van der Laan, Rev. Mod. Phys. 81, 235 (2009).

[2] P. Santini, R. Lémanski, and P. Erdos, Adv. Phys. 48, 537 (1999).

[3] C. Pfleiderer, Rev. Mod. Phys. 81, 1551 (2009).

[4] J. A. Mydosh and P. M. Oppeneer, Rev. Mod. Phys. 83, 1301 (2011).

[5] J. A. Mydosh and P. M. Oppeneer, Philos. Mag. 94, 3642 (2014).

[6] D. Belitz and T. R. Kirkpatrick, Phys. Rev. Lett. 119, 267202 (2017).

[7] H. Kotegawa, V. Taufor, D. Aoki, G. Knebel, and J. Flouquet, J. Phys. Soc. Jpn. 80, 083703 (2011).

[8] P. Gegenwart, Q. Si, and F. Steglich, Nat. Phys. 4, 186 (2008).

[9] T. Misawa, Y. Yamaji, and M. Imada, J. Phys. Soc. Jpn. 77, 093712 (2008).

[10] P. Nozieres and A. Blandin, J. Phys. (Paris) 41, 193 (1980).

[11] D. L. Cox, Phys. Rev. Lett. 59, 1240 (1987).

[12] C. Thomas, A. S. da Rosa Simoes, J. R. Iglesias, C. Lacroix, N. B. Perkins, and B. Coqblin, Phys. Rev. B 83, 014415 (2011).

[13] N. B. Perkins, M. D. Nunez-Regueiro, B. Coqblin, and J. R. Iglesias, Phys. Rev. B 76, 125101 (2007).

[14] I. Giannakis, J. Leshen, M. Kavai, S. Ran, C.-J. Kang, S. R. Saha, Y. Zhao, Z. Xu, J. W. Lynn, L. Miao, L. A. Wray, G.
Kotlira, N. Butch, and P. Aynajian, Sci. Adv. 5, eaaw9061 (2019).

[15] P. Wisniewski, A. Gukasov, and Z. Henkie, Phys. Rev. B 60, 6242 (1999).

[16] T. Durakiewicz, J. J. Joyce, G. H. Lander, C. G. Olson, M. T. Butterfield, E. Guziewicz, A. J. Arko, L. Morales, J. Rebizant, K. Mattenberger, and O. Vogt, Phys. Rev. B 70, 205103 (2004).

[17] J. Valenta, F. Honda, M. Vališka, P. Opletal, J. Kaštil, M. Míšek, M. Diviš, L. Sandratskii, J. Prchal, and V. Sechovský, Phys. Rev. B 97, 144423 (2018).

[18] P. S. Riseborough, B. Coqblin, and S. G. Magalhaes, Phys. Rev. B 85, 165116 (2012).

[19] B. Caroli, C. Caroli, and D. R. Fredkin, Phys. Rev. 178, 599 (1969).

[20] R. L. Stillwell, I.-L. Liu, N. Harrison, M. Jaime, J. R. Jeffries, and N. P. Butch, Phys. Rev. B 95, 014414 (2017).

[21] K. Shrestha, D. Antonio, M. Jaime, N. Harrison, D. S. Mast, D. Safarik, T. Durakiewicz, J.-C. Griveau, and K. Gofrik, Sci. Rep. 7, 6642 (2017).

[22] M. Valiska, H. Saito, T. Yanagisawa, C. Tabata, H. Amitsuka, K. Uhlirova, J. Prokleska, P. Proschek, J. Valenta, M. Misek, D. I. Gorbunov, J. Wosnitza, and V. Sechovsky, Phys. Rev. B 98, 174439 (2018).

[23] V. F. Correa, S. Francoual, M. Jaime, N. Harrison, T. P. Murphy, E. C. Palm, S. W. Tozer, A. H. Lacerda, P. A. 
Sharma, and J. A. Mydosh, Phys. Rev. Lett. 109, 246405 (2012).

[24] K. Haule and G. Kotliar, Europhys. Lett. 89, 57006 (2010).

[25] J. Schoenes, Phys. Rep. 66, 187 (1981).

[26] L. Havela, V. Sechovsky, F. R. de Boer, E. Bruck, and H. Nakote, Physica B (Amsterdam) 177, 159 (1992).

[27] S. Mašková, A. V. Andreev, Y. Skourski, S. Yasin, D. I. Gorbunov, S. Zherlitsyn, H. Nakotte, K. Kothapalli, F. Nasreen, C. Cupp et al., Phys. Rev. B 99, 064415 (2019).

[28] P. S. Riseborough, S. G. Magalhaes, and E. J. Calegari, Philos. Mag. 94, 3820 (2014).
[29] E. J. Calegari, S. G. Magalhaes, and P. S. Riseborough, npj Quantum Mater. 2, 48 (2017).

[30] N. Shah, P. Chandra, P. Coleman, and J. A. Mydosh, Phys. Rev. B 61, 564 (2000).

[31] P. M. Chaikin and T. C. Lubensky, Principles of Condensed Matter Physics (Cambridge University Press, Cambridge, UK, 1995).

[32] V. P. Mineev and M. E. Zhitomirsky, Phys. Rev. B 72, 014432 (2005).

[33] P. Chandra, P. Coleman, and R. Flint, Phys. Rev. B 91, 205103 (2015). 